\title{
Three-Dimensional Devices Transport Simulation Lifetime and Relaxation Semiconductor
}

\author{
Nouar Souad Fadila*, S. Mansouri**, M. Amrani**, P. Marie ${ }^{* * *}$, A. Massoum* \\ *Electrotechnics Department, Faculty of Technology, University Djilali Liabès of Sidi bel Abbès 22000 Algeria \\ ** Electronics Department, Faculty of Technology, University Djilali Liabès of Sidi bel Abbès 22000 Algeria \\ *** CIMAP UMR 6252CNRS-ENSICAEN-CEA-UCBN, 6 Boulevard du Marechal Juin, 14250 Caen Cedex France
}

\section{Article Info}

Article history:

Received Nov 8, 2014

Revised Dec 27, 2014

Accepted Jan 20, 2015

\section{Keyword:}

Gummel algorithm

Lifetime semiconductor

Newton algorithm

Relaxation semiconductor

Three-dimensional simulation

\begin{abstract}
Our work is to create a three-dimensional Simulator (3D) used for the study of the components to low geometry of design, and to determine in the volume structure the potential distributions and densities of free carriers in bias voltage given by solving the system of Poisson and two continuities equations. The initial version can simulate components of lifetime semiconductor. In this study, we make a comparison between the lifetime and relaxation semiconductor in the conduction mode. In order to create a larger Simulator, we'll perform a calculation by varying am bipolar lifetime way to move from lifetime semiconductor to relaxation semiconductor. We consider the case corresponding at two different values of diffusion lifetime $\tau_{0}$ which is corresponding to a measured lifetime in current transport. The method of resolution consists to linearization of the equations transport by the finite differences method. The algorithm for solving linear and strongly coupled equations deduced from the physical model is the Newton-Raphson. However, in order to allow a better convergence and consequently an improvement of time computing $3 \mathrm{D}$, a method combined, incorporating the Newton algorithm and the Gummel method was developed. PIN diodes are used for test of the simulation model.
\end{abstract}

Copyright (C) 2015 Institute of Advanced Engineering and Science. All rights reserved.

\section{Corresponding Author:}

A. Massoum,

Electrotechnics Department, Faculty of technology, University Djilali Liabès of Sidi bel Abbès, 22000 Algeria.

Email: ahmassoum@yahoo.fr

\section{INTRODUCTION}

To improve the SIM 3D software developed in our laboratory, have created a more general tool to calculate potential distributions and concentrations of free carriers, by a numerical solution of equations of transport for III - V semiconductors. Worth $\tau_{0}$, considerably higher than the dielectric relaxation time $\operatorname{\tau rd}\left(\tau_{\mathrm{rd}}\right.$ $<<\tau_{0}$ ), the results can be analyzed using simple injection and assuming a common unit holders in excess life expectancy which is constant for electrons and holes through the $v$ region this type of semiconductor will be called a semiconductor to life according to the terminology of Van Roosbroeck.

In the contrary case or $\tau_{0}$ is much lower than the dielectric relaxation time $\tau_{\mathrm{rd}}\left(\tau_{\mathrm{rd}}>>\tau_{0}\right)$ [1], the effects of space charge is very important by any and lifetimes of excess carriers vary greatly from one point to another along the structure. This type of semiconductor is called semiconductor to relaxation. This is the case of GaAs. The algorithm best comply with resolution nonlinear equations in partial differentials arising from the physical model, established is that of Newton, this method however converges rapidly if the entered values are properly chosen, we have therefore adapted a method combined, which brings both the method of Gummel and Newton, thus creating a link between the two algorithm sat the end for a reduction of computation time and a better convergence. 
We present in the order suitable mathematical model, as well as the physical model, numerical model followed by modeling results and their interpretation.

\section{THREE-DIMENSIONAL REPRESENTATION OF THE PHYSICAL EQUATIONS [2]-[3]}

In the stationary case and for the analysis of a homogeneous structure in three dimensions, the Basic (Fish and continuity) equations take the following form [4]-[5]

$$
\left\{\begin{array}{l}
\frac{\partial^{2} \psi}{\partial x^{2}}+\frac{\partial^{2} \psi}{\partial y^{2}}+\frac{\partial^{2} \psi}{\partial z^{2}}=\frac{q}{\varepsilon} \cdot\left(n-p+N_{D}^{+}-N_{A}^{-}-n_{r}\right) \\
\frac{1}{q} \cdot\left(\frac{\partial j_{n}}{\partial x}+\frac{\partial j_{n}}{\partial y}+\frac{\partial j_{n}}{\partial z}\right)=U \\
\frac{1}{q} \cdot\left(\frac{\partial j_{p}}{\partial x}+\frac{\partial j_{p}}{\partial y}+\frac{\partial j_{p}}{\partial z}\right)=-U
\end{array}\right.
$$

with

$$
\begin{aligned}
& U=U_{n}=U_{p}=\frac{n \cdot p-n_{1} \cdot p_{1}}{\tau p e \cdot\left(n+n_{1}\right)+\tau_{n e} \cdot\left(p+p_{1}\right)} \\
& j_{n}=q \cdot n \cdot \mu_{n} \cdot\left(\frac{\partial \psi}{\partial x}+\frac{\partial \psi}{\partial y}+\frac{\partial \psi}{\partial z}\right)+q \cdot D_{n} \cdot\left(\frac{\partial n}{\partial x}+\frac{\partial n}{\partial y}+\frac{\partial n}{\partial z}\right) \\
& j_{p}=q \cdot p \cdot \mu_{p} \cdot\left(\frac{\partial \psi}{\partial x}+\frac{\partial \psi}{\partial y}+\frac{\partial \psi}{\partial z}\right)-q \cdot D_{p} \cdot\left(\frac{\partial p}{\partial x}+\frac{\partial p}{\partial y}+\frac{\partial p}{\partial z}\right)
\end{aligned}
$$

with

- $\varepsilon=\varepsilon_{\mathrm{o}} . \varepsilon_{\mathrm{r}}$ The dielectric permittivity of the semiconductor

- $\varepsilon_{0}$ : The permittivity of vacuum

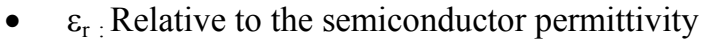

- $\psi$ : Electrostatique potentiel

- $\quad$ q: elementary charge $=1 \cdot 6 \cdot 10^{-19} \mathrm{C}$

- $\quad \mathrm{p}$ et $\mathrm{n}$ : free holes and electrons densities

- $\mathrm{N}_{\mathrm{D}}^{+}$et $\mathrm{N}_{\mathrm{A}}^{-}$: donors and acceptors ionized densities

- $\mathrm{n}_{\mathrm{r}}$ : The charge trapped on a deep centre. Where there are $\mathrm{n}$ deep centers be replaced $\mathrm{nr}$ by

$$
\sum_{i=1}^{n} n \text { ri }
$$

- $\quad \mathrm{j}_{\mathrm{n}}$ et $\mathrm{j}_{\mathrm{p}}$ : vector current densities of electrons and holes

- $\quad \mu_{\mathrm{n}}$ et $\mu_{\mathrm{p}}$ : mobilities of electrons and holes. $\mathrm{D}_{\mathrm{n}}$ et $\mathrm{D}_{\mathrm{p}}$ : Diffusion of électrons and holes constants

$$
\frac{D_{n}}{\mu_{n}}=\frac{K . T}{q} \quad \text { et } \quad \frac{D_{p}}{\mu_{p}}=\frac{K . T}{q}
$$

With

- $\mathrm{K}$ : BOLTZMANN constant

- $\mathrm{T}:$ absolue temperature.

- $\quad \mu_{\mathrm{n}}$ et $\mu_{\mathrm{p}}$ : mobilities of electrons and holes

Will be carrying loads in a material with a low density $\mathrm{N}_{R}$ centre combining the charge bomb space (-enr) is low compared with the space of free carriers and donors or ionized Acceptor charge.

Recombination plays an important role, it depends on the values of the parameters $\tau_{\mathrm{ne}}$ and $\tau_{\mathrm{pe}}$, the dielectric relaxation time $\tau_{\mathrm{rd}}$ and diffusion $\tau$ o life are important to analyze the behavior under conditions of non-balance of a given semiconductor 


$$
\begin{aligned}
& \tau_{r d}=\frac{\varepsilon}{\sigma_{e}}=\frac{\varepsilon}{e\left(n_{e} \mu_{n}+p_{e} \mu_{p}\right)} \\
& \tau_{o=} \frac{\tau_{n e\left(p_{e}+p_{t}\right)}+\tau_{p e\left(n_{e}+n_{t}\right)}}{\left(n_{e}+p_{e}\right)}
\end{aligned}
$$

These two characteristic times allow defining the two types of semiconductors: semiconductor lifetime or relaxation semiconductor has duration of life $\tau_{\mathrm{rd}}<<\tau_{0}$ Semiconductor a relaxation $\tau_{\mathrm{rd}}<<\tau_{0}$

\section{NUMERICAL MODEL}

First, we were interested to achieve a non-uniform mesh that establishes from the application of a calculation based on a geometric series of constant reason (a) with a variable size mesh. This mesh type can be coarse in neutral areas and fine in the neighboring regions of the $\mathrm{P}+$ interface [6]-[7]. The electrical parameters calculation at thermodynamic equilibrium is derived by applying the method of Gummel method also called decoupled method, [8]-[9]. Gummel method consists of a successive resolution of the three systems coupled $n$ equations in $\mathrm{N}$ unknowns. Each system of equations is dedicated to determine the value of a single type of unknown category. For example the Poisson equation provides the values for $\psi$ potential based on the concentrations of $\mathrm{N}$ and $\mathrm{P}$. The general principle of the Gummel method is as follows:

From an estimated initial solution $\left(\psi^{0}, \mathrm{~N}^{0}, \mathrm{P}^{0}\right)$, the equation $\mathrm{F} \psi(\psi, \mathrm{N}, \mathrm{P})=0$ unknown $\psi$ is firstly resolved. The values of thus determined will be carried forward in systems of equations $F_{n}$ et $F_{p}$. The equation $\mathrm{F}_{\mathrm{n}}(\psi, \mathrm{N}, \mathrm{P})=0$ is thus updated and resolved to turn the unknown $\mathrm{N}$. This update and resolution process is repeated alternately for $\mathrm{F}_{\psi}, \mathrm{F}_{\mathrm{n}}, \mathrm{F}_{\mathrm{p}}$ up to full convergence of the system, where:

$$
\left\{\begin{array}{l}
\frac{\partial^{2} \psi}{\partial x^{2}}+\frac{\partial^{2} \psi}{\partial y^{2}}+\frac{\partial^{2} \psi}{\partial z^{2}}-\frac{q}{\varepsilon} \cdot\left(n-p+N_{D}^{+}-N_{A}^{-}-n_{r}\right)=0=f_{\psi} \\
\frac{1}{q} \cdot\left(\frac{\partial j_{n}}{\partial x}+\frac{\partial j_{n}}{\partial y}+\frac{\partial j_{n}}{\partial z}\right)-U=0=f_{n} \\
\frac{1}{q} \cdot\left(\frac{\partial j_{p}}{\partial x}+\frac{\partial j_{p}}{\partial y}+\frac{\partial j_{p}}{\partial z}\right)+U=0=f_{p}
\end{array}\right.
$$

Gummel algorithm is represented by the following flowchart:

Following the electrical parameters at thermodynamic equilibrium calculations, these values will be injected into the Newton algorithm to calculate the same parameters under polarization, [10]-[11]-[12], is adding to each time one not for the polarization of $1 \mathrm{KT}$ /q (Figure 2). The application of Newton's method in numerical simulation of devices leads to solve simultaneously $\psi$, Fn, Fp. This is equivalent to calculate $\psi, \mathrm{N}$, $\mathrm{P}$ as a solution of a system to $3 \mathrm{~N}$ equations at each point of the network of three-dimensional discretization.

Three systems of equations discretized are grouped into a single system:

$$
\left(\begin{array}{l}
F_{\psi}(\Psi, N, P) \\
\mathrm{F}_{\mathrm{n}}(\Psi, N, P) \\
\mathrm{F}_{\mathrm{p}}(\Psi, N, P)
\end{array}\right)
$$

The stage of linearization extended to three systems $\mathrm{F}_{\psi}, \mathrm{Fn}, \mathrm{Fp}$ leads to solve the following system:

$\vec{u} . \vec{\delta}=-\vec{F}$, where: $\vec{u}$ : The complete Jacobian matrix of the system; $\vec{\delta}$ : The correction vector

This method also called method coupled it to reduce considerably the time of calculation, the fix was to deal with three-dimensional matrices or each element of the matrix is a square matrix of dimension $3 * 3$

$$
U=\left(\begin{array}{lll}
\frac{\partial F_{\psi}}{\partial \psi} & \frac{\partial F_{\psi}}{\partial N} & \frac{\partial F_{\psi}}{\partial P} \\
\frac{\partial F_{n}}{\partial \psi} & \frac{\partial F_{n}}{\partial N} & \frac{\partial F_{n}}{\partial P} \\
\frac{\partial F_{p}}{\partial \psi} & \frac{\partial F_{p}}{\partial N} & \frac{\partial F_{p}}{\partial P}
\end{array}\right) \quad \delta=\left(\begin{array}{c}
\delta_{\psi} \\
\delta_{n} \\
\delta_{p}
\end{array}\right)
$$




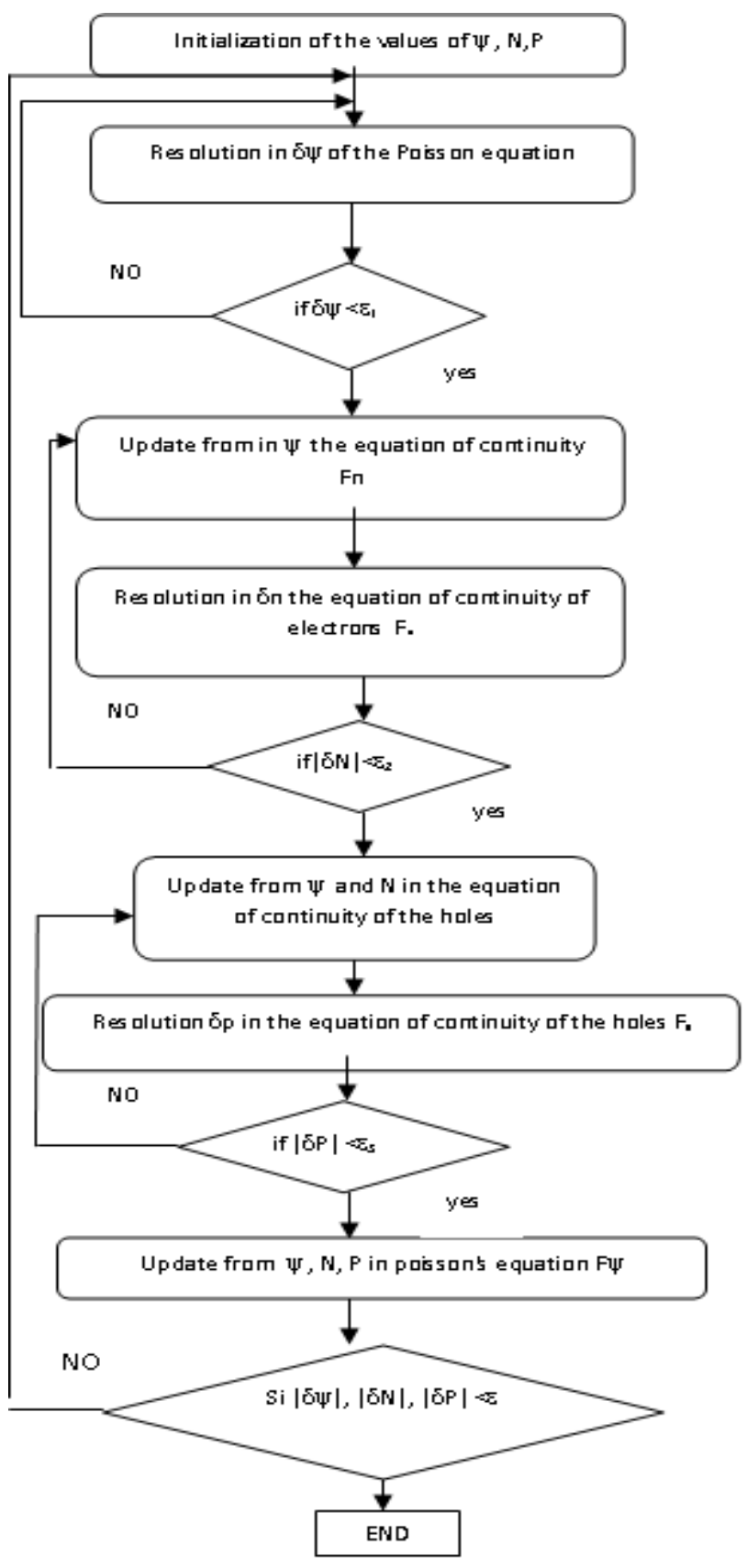

Figure 1. Resolution by the decoupled method chart

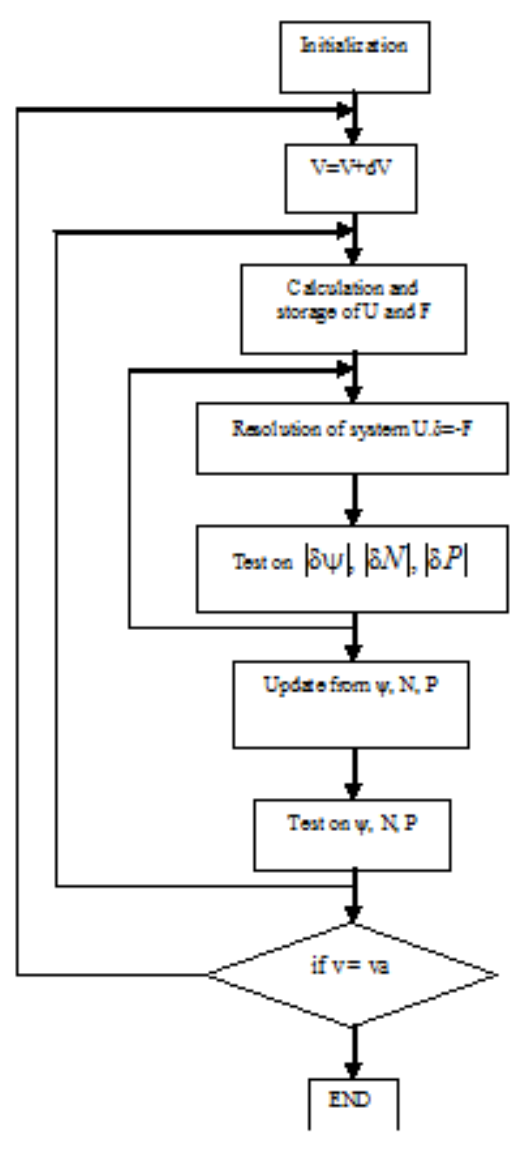

Figure 2. Organizational structure of resolution by the coupled method

Thus was created a new method combined the Gummel method and Newton's method using nonuniform mesh rather tight areas with loads of space and more coarse in neutral areas, with a considerable gain in time performance that turns long three-dimensional computation. 


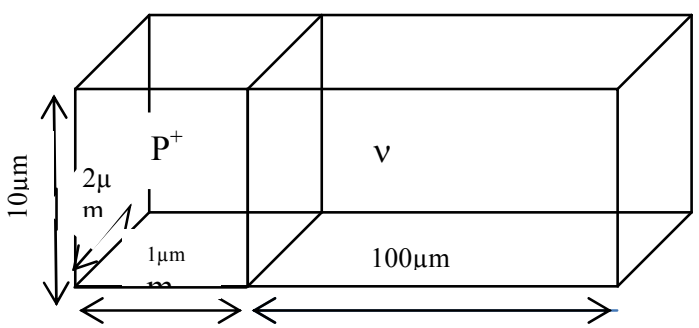

Figure 3. Structure used

The structure has been studied is a structure $\mathrm{p} v, v$ layer is slightly $\mathrm{N}$, and we treat the case of semiconductors to life and to relax, these opposite behaviors are obtained by adjusting settings $\tau_{\mathrm{ne}}$ et $\tau_{\mathrm{pe}}$, we will assume that the boundary conditions are such that the carrier concentrations are attached to their thermodynamic equilibrium values, and we will use two types of boundary conditions of Dirichlet on unknown plans.

\section{RESULTS AND INTERPRETATION}

Digital simulation software is written in $\mathrm{C}++, \mathrm{DEV} \mathrm{C}++$. Thermodynamic equilibrium study is an essential step for a first simulation of a test structure. Furthermore, the sensitivity of Newton's method to the initial values will lead us to use results from the thermodynamic equilibrium, as initial values. In addition the thermodynamic Equilibrium State can give us a first vision of the distributions of potential and free carriers through a junction [13]

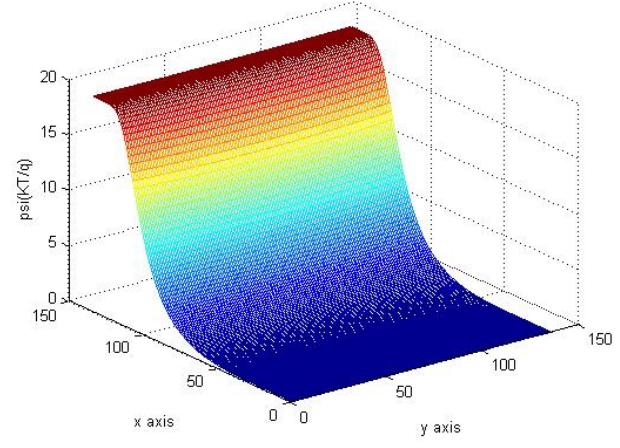

Figure 4. Profile potential distribution at thermodynamic equilibrium

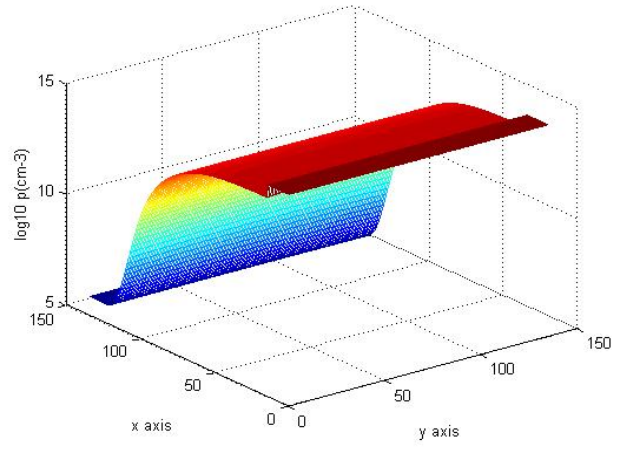

Figure 5. Profile holes distribution at thermodynamic equilibrium

Thermodynamic equilibrium simulation results are obtained by application of the algorithm called gummel as decoupled method results for the SC to life is the same for the sc to relaxation and they comply with the physical parameters given to the $\mathrm{p}$ region $=3.10^{14} \mathrm{~cm}^{-3}$ and the region $v \mathrm{Nd}=1.5 .10^{11} \mathrm{~cm}^{-3}$ to better see it will represent these results following the $\mathrm{x}$-axis only

It is visible that space charge zone extends mainly through the largest structure through the less doped structure $\mathrm{N}$ may has the consequence that conduction regime will be controlled by an effect of contact $\mathrm{P} v$, or diffusion potential is $20 \mathrm{KT} \backslash \mathrm{q}$ conformed of analytical calculation:

$\mathrm{vd}=\log \left(\left(\mathrm{N}_{\mathrm{A}} * \mathrm{~N}_{\mathrm{d}}\right) /(\right.$ ni*ni) $),[14]$

By digital application found

$\mathrm{V}_{\mathrm{d}}=20.03 \mathrm{KT} / \mathrm{q}$ 


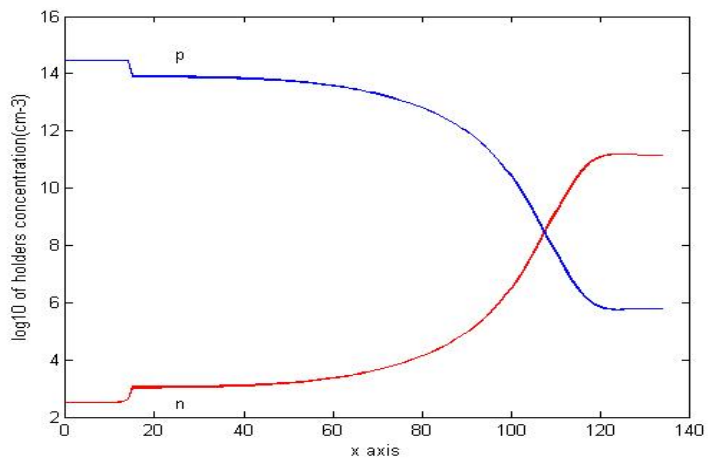

Figure 6. Profile holders distribution at thermodynamic equilibrium

The appearances of curves as well as the orders of magnitude of different concentrations determined by calculating 3D are physically correct.

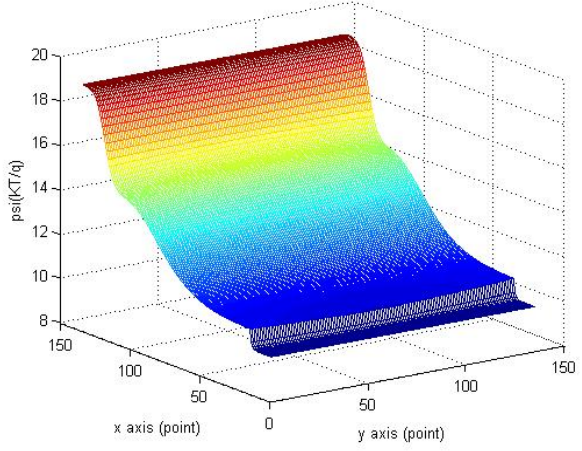

Figure 7. Profile of the distribution potential for a polarization of $10 \mathrm{KT} \backslash \mathrm{q}$ for a lifetime SC

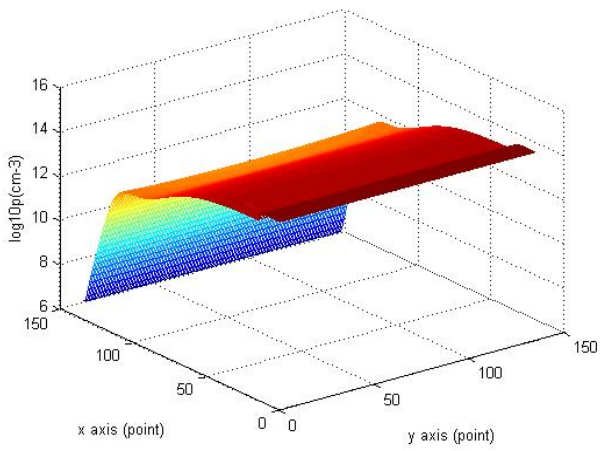

Figure 9. Profile of the density distribution of the holes for a polarization of $10 \mathrm{KT} \backslash \mathrm{q}$ for lifetime SC

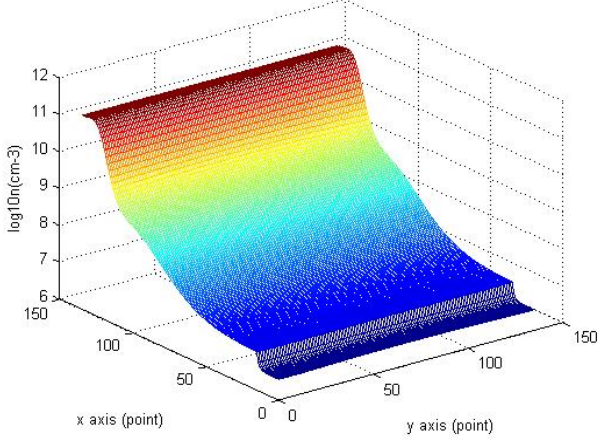

Figure 8. Profile of the density distribution of electrons for a polarization of $10 \mathrm{KT} \backslash \mathrm{q}$ for a lifetime SC

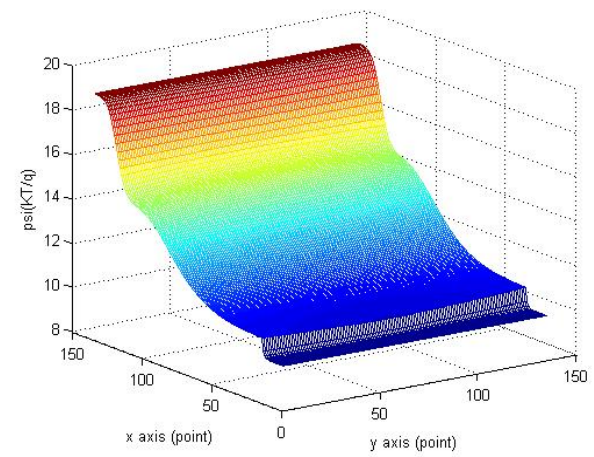

Figure 10. Profile of the density distribution of the electrons for a polarization of $10 \mathrm{KT} \backslash \mathrm{q}$ for relaxation SC 


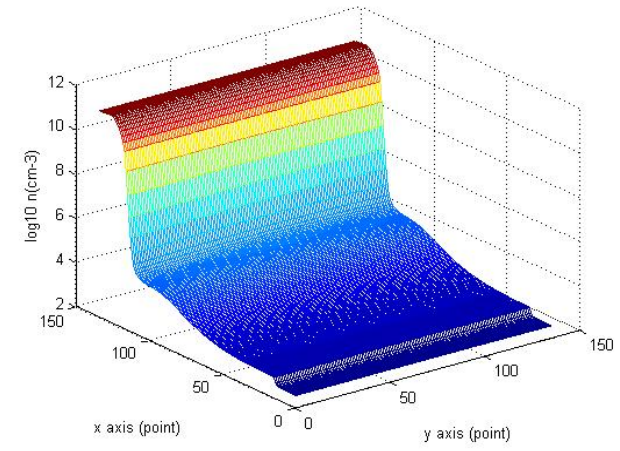

Figure. 11 Profile of the density distribution of electrons for a polarization of $10 \mathrm{KT} \backslash \mathrm{q} \mathrm{SC}$

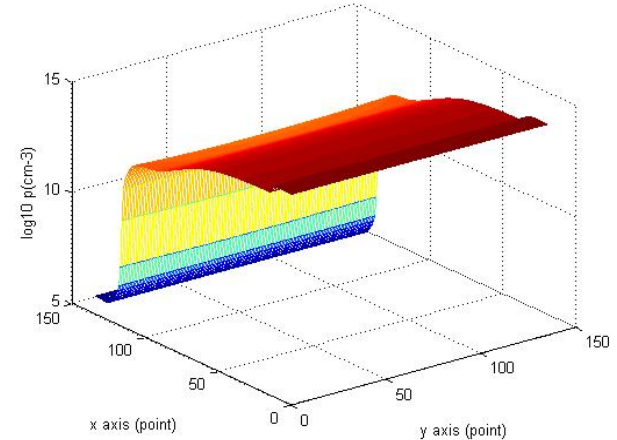

Figure 12. Profile of the density distribution for a relaxation of the holes for a polarization of $10 \mathrm{KT} \backslash \mathrm{q}$ for a relaxation $\mathrm{SC}$

Plots are given according to mesh point and not by length; the $\mathrm{P}^{+}$region is discretized on 15 points and region $\mathrm{N}$ on 135 points, thus allowing to better presenting the space charge zone. The plot are given according to mesh points and not by the length, $\mathrm{P}^{+}$region is discretized on 15 points and the $\mathrm{N}$ on 135 points, allowing better present area of space charge.

In order to enable comparisons between different distribution curves of free carriers in different polarizations, and the ability to draw in the same graph, we chose to draw in $\mathrm{x}$ - axis

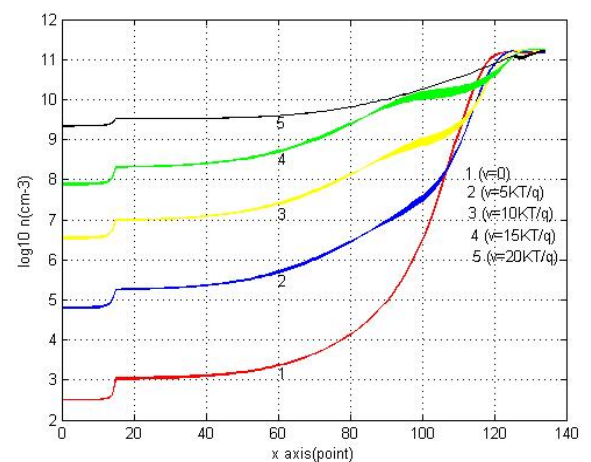

Figure 13. Profile of the density distribution of electrons for a lifetime SC

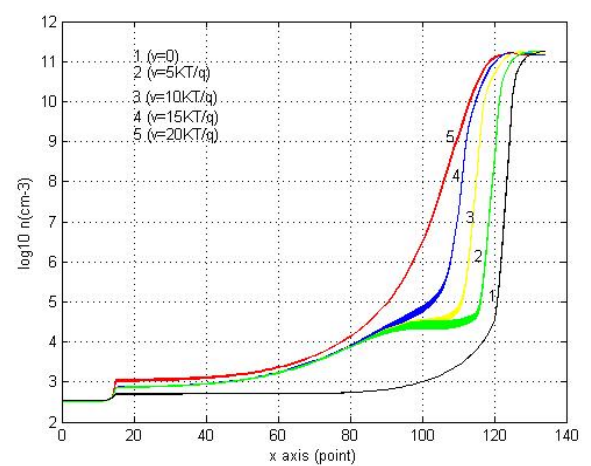

Figure 15. Profile of the density distribution of electrons for a relaxation $\mathrm{SC}$

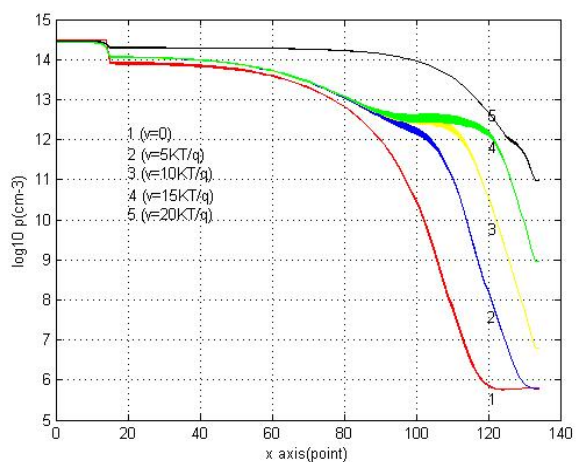

Figure 14. Profile of the density distribution of the holes for a lifetime SC

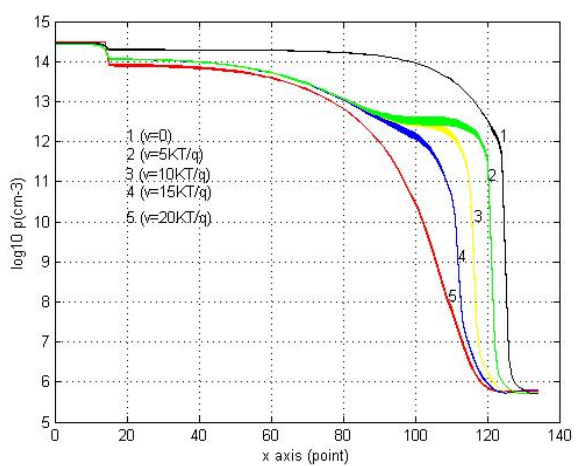

Figure 16. Profile of the density distribution of the holes for a relaxation $\mathrm{SC}$ 


\section{INTERPRETATION OF RESULTS}

All results found under polarization are obtained by application of the Newton algorithm. The structure is equipped with only one contact injecting. For the lifetime semiconductor under polarization note a reduction in the width of the area of space charge from equilibrium thermodynamics but widely more extensive than that of the relaxation semiconductor.

The extension of the area of space in the region $v$ charge derives essentially to free carriers and ionized donor, note that throughout the structure for a lifetime semiconductor

$\Delta \mathrm{n}=\Delta \mathrm{p}$, the trend is towards the neutralization of the space charge, the values of $\mathrm{n}$ and $\mathrm{p}$ in neutral zones increase checking the equation

$$
n=n_{0} \exp \left(\frac{e v}{K T}\right) \quad p=p_{0} \exp \left(\frac{e v}{K T}\right)
$$

For relaxation semiconductor for low polarization, the values of $\mathrm{n}$ and $\mathrm{p}$ in neutral zones keeps the same values as that of thermodynamic equilibrium, for voltages lower than $20 \mathrm{KT} \backslash \mathrm{q}$ the conduction is controlled by the effect of contact if $\mu \mathrm{n}>\mu \mathrm{p}$, the front of recombination occurs in neighborhood of the contact $\mathrm{P}$ the right of the front of recombination we observed depletion of holes. For voltages in order to 20 $\mathrm{KT} \backslash \mathrm{q}$ is reached almost flat bands regime, there is the same remarks and an increase in the density of electrons and holes in $\mathrm{P}$ side and $v$ side respectively, unlike the relaxation semiconductor, where we note a front of recombination over near the $\mathrm{P}$ region.

\section{CONCLUSION}

The purpose of our study is the comparison of two semiconductors having the same electrical parameters except the relaxation time dielectric, for the lifetime semiconductor $\tau_{\mathrm{nt}}=\tau_{\mathrm{pt}}=10^{-8} \mathrm{~s}$ and a relaxation semiconductor $\tau_{\mathrm{nt}}=\tau_{\mathrm{pt}}=10^{-11} \mathrm{~s}$ with a $\tau_{\mathrm{rd}}=4.810^{-9} \mathrm{~s}$. For the $v$ Zone this adjustment leads to a different behavior for the two semiconductors types. We also note a great difference between the semiconductor Relaxation and the insulators whose free-carrier concentration is negligible.

\section{REFERENCES}

[1] R. Ardebili, J.C. Nathalie. Study by numerical simulation of transport in semiconductors in the presence of center deep. Thesis PhD Centre Montpellier electronics, (1992)

[2] H. Mathieu. Physics of semiconductors and electronic components Courses and exercises corrected. 6th edition Masson, Paris 2009.

[3] F.S. Nouar. Three-dimensional modeling of transport in PN junctions in the presence of deep centers. Thesis of University of Djilali Liabès, Sidi Bel Abbès, 2002

[4] J.D. Chatelin. Dispositifs of semiconductor. Edition Georgi, 1979

[5] R. Menezla. CLAC 3D program three-dimensional resolution of Poisson's equation. Thesis Phd, Ecole Centrale de Lyon, no 85-05, 1990

[6] Jean-Pierre Corriou. Numerical methods and optimization: theory and practice for the engineer. Édition Paris tec et doc, 2010

[7] M. Kemp, C.G. Tannous, M. Meunier. Amorphous silicon device simulation by an adapted Gummel method. IEEE transation on electron devices, Pergamon press, vol 27, no 4, pp.319-328, 1987

[8] O.E. Akcasu. Convergence proprieties of Newton's methode for the solution of the semiconductor transport equations and hybrid solution techniques for multidimensional simulation of VLSI devices Solid state electronics. Pergamon press, vol 27. no 4, pp 349-328,1987.

[9] M. Khadraoui. Use of coupled and decoupled methods for three-dimensional simulation of devices at junction PN. Thesis, University Djillali Liabès, Sidi Bel Abbè, 1998

[10] A. Resfa. 3D modeling of this breakdown by avalanche in the structures of the PN junction of GaAs insulating semi junction presented centers deep. Thesis, University of Djilali Liabès, sidi bel Abbès, 2005

[11] C.S. Rafferty, M.R. Pinto and W. Dutton. Iterative methods in semiconductors device simulation. IEEE transation on electron devices, vol ED-32 no 100, pp. 2018-2027, October 1985

[12] Bouabddellah Badra. Simulation numerical of conduction in the volume of components to semiconductors to relaxation. Thesis PhD Faculty of engineer science, University Sidi Bel Abbès Algeria, 2004

[13] Claude Delannoy. C++ for C programmers. Edition Eyrolles, paris 2007

[14] A. Benchiheb. Modélisation d'un transistor bipolaire de puissance. Thèse de magister université de Constantine, 1996 